\title{
Nitrogen Dioxide Sterilization
}

National Cancer Institute

\section{Source}

National Cancer Institute. Nitrogen Dioxide Sterilization. NCI Thesaurus. Code C107416.

A sterilization process that uses nitrogen dioxide to kill microorg anisms. 